\title{
A FIELD-TRIAL OF TWO RESTORATIVE MATERIALS USED WITH ATRAUMATIC RESTORATIVE TREATMENT IN RURAL TURKEY: 24-MONTH RESULTS
}

\author{
Ertugrul ERCAN' ${ }^{1}$, Ç. Türksel DÜLGERGIL ${ }^{1}$, Mübin SOYMAN² $^{2}$, Mehmet DALLI $^{3}$, Isil YILDIRIM
}

1- Assistant Professor, Kýrýkkale University Dental School, Department of Operative Dentistry, Kýrýkkale, Turkey.

2- Professor and Head, Operative Dentistry in Dental School of Yeditepe University, Istanbul, Turkey.

3- DDS Dr. Dicle University Dental School, Department of Operative Dentistry, Diyarbakýr, Turkey.

4- DDS, Kýrýkkale University Dental School, Department of Operative Dentistry, Kýrýkkale, Turkey.

Corresponding address: Ertugrul ERCAN - University of Kirikkale Dental School - Department of Operative Dentistry - 71000 Kirikkale - Turkey Phone: +903182244927 - Fax: +90 3182244683 - e-mail:ertugrulercan@ hotmail.com

Received: July 02, 2008 - Accepted: November 09, 2008

\begin{abstract}
$O$

bjective: The purpose of this study was to investigate the clinical performance of high-strength glass ionomer cement (HSGIC) and resin-modified glass ionomer (RMGIC) in single and multiple surface carious cavities in the field conditions. Material and Methods: A split-mouth design, including ninety-one fillings placed on contra lateral molar pairs of 37 children, was used in permanent dentition. As filling materials, a HSGIC (Ketac Molar/3M ESPE) and a RMGIC (Vitremer/ 3M ESPE) were used with the Atraumatic Restorative Treatment (ART). Baseline and 6, 12 and 24-month evaluations of the fillings were made with standardART and USPHS criteria by two examiners with kappa values of 0.92 and 0.87 for both criteria. Results: According to the USPHS criteria, the retention rates of RMGIC and HSGIC restorations were $100 \%$ and $80.9 \%$ for single surface, and $100 \%$ and $41.2 \%$ for multiple surface restorations after 24 months, respectively. Irrespective of surface number, RMGIC was significantly superior to HSGIC ( $\mathrm{p}=0.004$ ), according to both standard-ART and USPHS criteria. Conclusion: The results indicate that RMGIC may be an alternative restorative technique in comparison to high-strength GIC applications in ART-field-trials. However, further clinical and field trials are needed to support this conclusion.
\end{abstract}

Key words: Atraumatic Restorative Treatment. ART. Glass-ionomer cements. Resin-modified glass-ionomer. Light Emitted Diode (LED).

\section{INTRODUCTION}

A new method for treating dental caries that involves no drill, running water or electricity was presented at the headquarters of the World Health Organization on World Health Day in $1994{ }^{12}$. This approach, called "Atraumatic Restorative Treatment" (ART) consists of manually cleaning dental cavities with hand instruments and restoring them with an adhesive fluoride-releasing material ${ }^{1}$. Since that time, ART has been developed into a reliable alternative to restorations performed even at well-equipped dental clinics $^{2,18,29}$. This technique involves removal of only carious tooth structures with hand instruments, and the use of glass ionomer cements (GICs) as filling material ${ }^{12}$.

In spite of the fact that first results of ART approach were more promising, especially with single-surface restorations after 1 year, material loss (wear/dissolution) was more common in GIC material in the following years ${ }^{15,21,22,26}$. Subsequently, some improvements have been performed to increase the resistance and manipulation of GICs due to their moisture sensitivity and low wear resistance ${ }^{28}$.

Although conventional GICs have relatively poor mechanical and adhesive strengths, their satisfactory biological features, ease of use, and low costs are distinct advantages $^{26}$. Further development of GICs focused on a higher-powder-to-liquid ratio, and smaller glass particles leading to high viscosity. Therefore, more strength GICs (non-metal-reinforced: Fuji IX, Ketac Molar; metalreinforced: Hi Dense), which should be packable as amalgam was found, and revealed enhanced flexural strength characteristics $^{14,17}$.

When compared to early field trials, newer ART studies using specifically marketed GIC material have shown promising results for the restoration of single-surface caries lesions in permanent teeth ${ }^{11}$. Although the success rate reported by most studies is approximately $90 \%$ for single surface restorations in permanent dentition, the cumulative survival percentage seems considerably variable according 
to evaluation periods, materials and geography: $66.1 \%$ for 6 years in Syria $^{13} ; 72.3 \%$ for 6 years in Tanzania ${ }^{22} ; 92.5$ to $94.9 \%$ for two years in Latvia ${ }^{29} ; 93.3 \%$ for two years in Filipines $^{24}, 76 \%$ and $59 \%$ of the small and large restorations for six years in $\mathrm{China}^{19}$; and $66 \%$ for two years in $\mathrm{Mexico}^{20}$. On the other hand, the success rates have been much lower for multi-surface molar restorations (55\% to $75 \%$ ), and found to be closely related with the operator, the materials used, and the ART method of cavity preparation ${ }^{10}$.

Materials have recently led to varied alternatives to ART field trials ${ }^{7,9,10}$. Of these, light-curing materials, such as resin modified glass ionomers (RMGIC), which can easily be cured in rural conditions by a rechargeable curing device, a light emitting diode (LED), may be an alternative to GICs in non-electrical conditions ${ }^{7}$.

The objective of this 24-month-follow-up study was to evaluate, using the United States Public Health Service (USPHS) and ART criteria, the in vivo performance of two restorative materials - one HSGIC and one RMGIC - cured with LED in field conditions. The null hypothesis was that there is no difference in the success rate between restorations placed using the ART approach using either GIC or RMGIC after two years.

\section{MATERIAL AND METHODS}

This study was conducted in rural districts of the city of Diyarbakir in Turkey. The target group consisted of 7-to12-year-old children with dental caries in permanent teeth. Their oral hygiene was very low, and dft and DMFT were 5.2 and 1.8, respectively. The selected children and their parents were informed of the nature and procedures of this study by the school authorities. The voluntary nature of participation was explained, and parental consent was obtained. Parental consent was obtained in writing through the school authorities. The study protocol was approved by the National Educational Management of Diyarbakýr (2002).

Thirty-seven children who had one or two bilateral matched pairs of carious posterior permanent teeth that required either Class I or Class II restorations were selected. In this procedure, split-moth approach was employed for the allocation of the two restorative materials. Intraoral assignment of the material to the teeth was done by the loss of the coin as seen in Mandari, et al. ${ }^{23}$ (2001).

A random number table was used to determine which tooth of a pair was to be restored with either the control [HSGIC - Ketac Molar (3M ESPE, St Paul, MN, USA)] or the test [RMGIC - Vitremer (3M/ESPE Dental Products, St. Paul, MN, USA)] material.

One experienced dentist performed all treatment procedures during 2 months in school premises (ÇTD). He had previously trained in a previous ART field-trial for 4 years in the same region. Another trained dentist assisted him (EE). The placement of ART fillings closely followed the procedures described by Frencken, et al. ${ }^{12}$ (1996). When preparing a carious tooth to receive an ART restoration, the cavity entrance was widened, if required, with a small hatchet before the removal of soft caries with sharp spoon excavators. Prior to being filled, the prepared cavity and adjacent enamel were cleaned with a dentine conditioner, which was the mixing liquid supplied with the glass ionomer cement itself. The liquid was removed after 10-15 s with a wet cotton pellet and the cavity was then blotted dry. The glass ionomer cement was mixed by hand according to the manufacturer's instructions, and applied to the cavities with the "press finger" technique. A petroleum jelly layer was applied over the finished restorations. RMGIC material was applied as another restorative option using the ART technique as follows; (i) the operated quadrant was isolated with cotton-wool rolls, (ii) after cleaning the tooth surface and widening the entrance of the lesion, soft dentinal caries was firstly removed from the floor of the cavity, and secondly from the dentin-enamel junction. If the carious lesion extended deep into the dentin, a thin layer of a calcium hydroxide liner was carefully placed in the bottom of the cavity, (iii) the surface, including dentinal tissue, was conditioned with a conditioning-solution (Vitremer ${ }^{\mathrm{TM}}$ Primer/ 3M ESPE) provided by the manufacturer, using an adequate-sized moistened cotton pellet, (iv) the material was mixed according to the manufacturer's instructions with the proportion of 2 to 3 powder measures for every liquid drop. Then, newly mixed material was squeezed into the cavity. (v) Before light-curing, to avoid air bubbles, to adapt the material into the cavity, and to remove excessive material, initial finger pressing coated with petroleum jelly was performed, and a slight pressure was applied. (vi) Since the material would be too hard to be trimmed after curing, an occlusion adjustment was made just before curing, and to provide a smooth surface, finger pressing was repeated before curing. (vii) For adequate polymerization, at least 30 s of light-curing with LED (Light Emitting Diyote - Elipar Freelight ${ }^{\mathrm{TM}} / \mathrm{ESPE}$, Germany) was applied. (viii) To eliminate surface roughness, a hatchet was used with carvingmotion. Finally, a surface protector solution provided by the manufacturer was applied and cured.

The restorations were evaluated in accordance with both USPHS and ART score systems at baseline, 6, 12 and 24 months (Figures 1 and 2). For ART rating procedures, the evaluations were carried out in the children's own school environment by two calibrated dentists, using a ball-endedWHO probe. Baseline evaluation of the restorations took place one week later. Two blinded experienced dentists (MD and IY), evaluated the restorative materials at baseline, 6 , 12 and 24-month periods. Evaluation consistency was assessed in a sample of 30 restorations (15 RMGIC and 15 GIC) in 12 children at baseline.

For the USPHS-scoring procedure, a sharp explorer was used. The mean values of Cohen's kappa for ART and USPHS criteria (examiner 1 vs. 2) were 0.92 and 0.87 , indicating good inter-examiner agreement. The basic disagreement was usually seen in the presence and degree of marginal discoloration of RMGIC restorations for USPHS criteria. According to the ART score system, a restoration was assessed as successful if it was recorded as score 0,1 


\begin{tabular}{|c|c|c|}
\hline Category & Scores & Criteria \\
\hline \multirow[t]{2}{*}{ Retention } & Alpha & No loss of restorative material \\
\hline & Charlie & Any loss of restorative material \\
\hline \multirow[t]{3}{*}{ Color Match } & Alpha & Matches tooth \\
\hline & Bravo & Acceptable mismatch \\
\hline & Charlie & Unacceptable mismatch \\
\hline \multirow[t]{3}{*}{ Marginal Discoloration } & Alpha & No discoloration \\
\hline & Bravo & Discoloration without axial penetration \\
\hline & Charlie & Discoloration with axial penetration \\
\hline \multirow[t]{2}{*}{ Secondary Caries } & Alpha & No caries present \\
\hline & Charlie & Caries present \\
\hline \multirow[t]{3}{*}{ Anatomic Form } & Alpha & Continuous \\
\hline & Bravo & Slight discontinuity, clinically acceptable \\
\hline & Charlie & Discontinuous, failure \\
\hline \multirow[t]{3}{*}{ Marginal Adaptation } & Alpha & Closely adapted, no detectable margin \\
\hline & Bravo & Detectable margin clinically acceptable \\
\hline & Charlie & Marginal crevice clinical failure \\
\hline \multirow[t]{3}{*}{ Surface Texture } & Alpha & Enamel like surface \\
\hline & Bravo & Surface rougher than enamel, clinically acceptable \\
\hline & Charlie & Surface unacceptable rough \\
\hline
\end{tabular}

FIGURE 1- Criteria used to evaluate USPHS (1)

\begin{tabular}{|c|c|}
\hline SCORE & ASSESSMENT CRITERIA \\
\hline 0 & Present, good \\
\hline 1 & $\begin{array}{l}\text { Present, slight marginal defect for whatever reason, at any one place which is less than } 0.5 \mathrm{~mm} \text { in depth: no } \\
\text { repair is needed }\end{array}$ \\
\hline 2 & $\begin{array}{l}\text { Present, marginal defect for whatever reason, at any one place which is deeper than } 0.5 \mathrm{~mm} \text {, but less than } \\
1.0 \mathrm{~mm} \text { : repair is needed }\end{array}$ \\
\hline 3 & Present, gross defect of more than $1.0 \mathrm{~mm}$ in depth: repair is needed \\
\hline 4 & Not present, restoration has ( almost) completely disappeared: treatment is needed \\
\hline 5 & Not present, other restorative treatment has been performed \\
\hline 6 & Not present, tooth has been extracted \\
\hline 7 & $\begin{array}{l}\text { Present, wear and tear gradually over larger parts of the restoration but is less than } 0.5 \mathrm{~mm} \text { at the deepest } \\
\text { point : no repair is needed }\end{array}$ \\
\hline 8 & $\begin{array}{l}\text { Present, wear and tear gradually over larger parts of the restoration which is deeper than } 0.5 \mathrm{~mm} \text { : repair is } \\
\text { needed }\end{array}$ \\
\hline 9 & Unable to diagnose \\
\hline
\end{tabular}

0, 1 and $7=$ =successful; 2, 3, 4 and 8=failure; 5, 6 9=excluded

FIGURE 2- Clinical criteria used to evaluate ART restorations (6)

or $7^{1}$.

All data were analyzed using the GraphPad Prism V.3 PC software (GraphPad Software Inc., San. Diego CA). For testing differences in both RMGIC and GIC groups between baseline, 6-month, 12-month, and 24-month results, Mc Nemar's test was used with significance set level at $\mathrm{p}<0.05$.
To evaluate the differences between RMGIC and GIC groups, and also between single and two surface restorations, Chi-square test was also used with significance level set at $\mathrm{p}<0.05$. 
TABLE 1- ART scoring number and percent distribution of both RMGI and GIC-ART restorations at baseline (Scores 0 and 7 are successful) and comparison of two materials $(p<0.05)$

\begin{tabular}{|c|c|c|c|c|c|}
\hline \multirow{2}{*}{\multicolumn{2}{|c|}{ Art Scoring }} & \multicolumn{2}{|c|}{ RMGI } & \multicolumn{2}{|c|}{ GIC } \\
\hline & & One surface & Two surfaces & One surface & Two surfaces \\
\hline Baseline & 0 & $31(100 \%)$ & $21(100 \%)$ & 22 (100\%) & $17(100 \%)$ \\
\hline \multirow{6}{*}{ 6-Month } & 0 & $30(96.8 \%)$ & $18(85.7 \%)$ & $18(81.8 \%)$ & 5 (29.4\%) \\
\hline & 1 & $1(3.2 \%)$ & $3(14.3 \%)$ & $3(13.6 \%)$ & $9(52.9 \%)$ \\
\hline & 2 & & & & $1(5.9 \%)$ \\
\hline & 3 & & & & $1(5.9 \%)$ \\
\hline & 4 & & & & $1(5.9 \%)$ \\
\hline & 7 & & & & $1(4.5 \%)$ \\
\hline \multirow[t]{6}{*}{ 12-Month } & 0 & $29(100 \%)$ & 15 (71.4\%) & $16(76.2 \%)$ & $4(23.5 \%)$ \\
\hline & 1 & & $6(28.6 \%)$ & $2(9.5 \%)$ & $4(23.5 \%)$ \\
\hline & 2 & & & $2(9.5 \%)$ & $4(23.5 \%)$ \\
\hline & 3 & & & & $1(5.9 \%)$ \\
\hline & 4 & & & & $2(11.8 \%)$ \\
\hline & 7 & & & $1(4.8 \%)$ & $2(11.8 \%)$ \\
\hline \multirow{6}{*}{ 24-Month } & 0 & 29 (100\%) & $15(71.4 \%)$ & $15(71.4 \%)$ & $2(11.8 \%)$ \\
\hline & 1 & & $6(28.6 \%)$ & $2(9.5 \%)$ & $3(17.6 \%)$ \\
\hline & 2 & & & $2(9.5 \%)$ & $3(17.6 \%)$ \\
\hline & 3 & & & $2(9.5 \%)$ & $1(5.9 \%)$ \\
\hline & 4 & & & & $6(35.3 \%)$ \\
\hline & 7 & & & & $2(11.8 \%)$ \\
\hline
\end{tabular}

\section{RESULTS}

A total of 91 restorations were placed in permanent teeth. Fifty-two were restorations with RMGIC and 39 were restorations with HSGIC, in that 21 and 17 had two surfaces, respectively. At baseline, 91 restorations (37 children) were assessed. Out of 91 restorations, 3 (two with RMGIC, one with HSGIC) were lost for follow-up over the 24-month period. While all restorations were present in the RMGIC group, a total of 6 (all in the two-surface group) HSGICrestorations were lost after 24 months (according to "retention" criteria of the USPHS- criteria system). The difference was statistically significant $(\mathrm{p}=0.004)$.

With respect to recurrent caries and/or the occurrence of new caries between teeth filling interfaces, all restorations in both groups were assessed as perfect at baseline, 6 and 12-month study periods, but not at 24 months. A total of 8 restorations in the HSGIC group showed the occurrence of new caries between tooth restoration interfaces in this recall period. The difference between both groups was statistically significant $(\mathrm{p}=0.002)$

The percentage and number distribution of RMGIC and HSGIC groups with respect to the ART scoring system is presented in Table 1 for both single- and- multiple-surface restorations, separately, and Figure 3. Irrespective of the surface number, $100 \%$ of RMGIC and $63.2 \%$ of HSGIC restorations were classified as successful after 24 months. The difference was statistically significant $(p=0.0001)$. In the comparison of single- and - multiple-surface-RMGIC restorations after 6,12 and 24 months, a statistically difference was observed at both 12 and 24 months (for both observation periods, $\mathrm{p}<0.002$ ). As a general rule, singlesurface restorations had a significantly better clinical performance than did multiple-surface restorations, especially for the HSGIC group.

When comparing both groups in each study period, a clear difference was observed in both $12(\mathrm{p}=0.05)$ and 24month $(p=0.02)$ results for single-surface restorations. However, a statistically significant difference was definitely found in either observation period for multiple-surface restorations (for 6 months, $\mathrm{p}<0.011$; for 12 months, $\mathrm{p}<0.008$; and for 2 months, $\mathrm{p}<0.0001)$.

Distributions of the retained RMGIC and HSGIC restorations among the USPHS criteria, including marginal discoloration, anatomic form, and marginal adaptation, are shown in Table 2. In the overall results, multiple-surface restorations were worse than single-surface restorations for both groups according to marginal discoloration. When comparing both groups, the statistical difference was only found at 24 months for surface restorations, and it was in favor of the RMGIC group (Figure 4).

A statistically significant difference in the loss of marginal adaptation between RMGIC and HSGICrestorations at all evaluation periods for multiple- surface (see Table 2; at 6 months, $\mathrm{p}<0.04$; at 12 months, $\mathrm{p}<0.03$; at 24 months, $\mathrm{p}<0.007$ ), but not for single-surface groups. 
TABLE 2- Number and percent distribution of both RMGI and GIc-ART restorations at baseline, 6, 12 and 24-month-recall according to USPHS criteria

\begin{tabular}{|c|c|c|c|c|c|}
\hline \multicolumn{2}{|c|}{ Anatomic Form } & \multicolumn{2}{|c|}{ RMGI } & \multicolumn{2}{|c|}{ GIC } \\
\hline & & One surface & Two surfaces & One surface & Two surfaces \\
\hline \multirow[t]{2}{*}{ Baseline } & $A$ & $31(100 \%)$ & $21(100 \%)$ & $17(77.3 \%)$ & $13(76.5 \%)$ \\
\hline & B & & & $5(22.7 \%)$ & $4(23.5 \%)$ \\
\hline \multirow[t]{3}{*}{ 6-Month } & $A$ & $29(93.5 \%)$ & $16(76.2 \%)$ & $11(50 \%)$ & $10(58.8 \%)$ \\
\hline & B & $2(6.5 \%)$ & $5(23.8 \%)$ & $10(45.5 \%)$ & $4(23.5 \%)$ \\
\hline & C & & & $1(4.5 \%)$ & $3(17.6 \%)$ \\
\hline \multirow{4}{*}{ 12-Month } & $A$ & $29(93.5 \%)$ & $11(52.4 \%)$ & $10(47.6 \%)$ & $5(29.4 \%)$ \\
\hline & $\mathrm{B}$ & $2(6.5 \%)$ & $10(47.6 \%)$ & $8(38.1 \%)$ & $6(35.3 \%)$ \\
\hline & $\mathrm{C}$ & & & $3(14.3 \%)$ & $4(23.5 \%)$ \\
\hline & $\mathrm{D}$ & & & & $2(11.8 \%)$ \\
\hline \multirow[t]{4}{*}{ 24-Month } & $A$ & $29(93.5 \%)$ & $10(47.6 \%)$ & $10(47.6 \%)$ & $5(29.4 \%)$ \\
\hline & $\mathrm{B}$ & $2(6.5 \%)$ & $10(47.6 \%)$ & $8(38.1 \%)$ & $3(17.6 \%)$ \\
\hline & $\mathrm{C}$ & & $1(4.8 \%)$ & $3(14.3 \%)$ & $4(23.5 \%)$ \\
\hline & $\mathrm{D}$ & & & & $5(29.4 \%)$ \\
\hline \multicolumn{2}{|c|}{ Marginal } & \multicolumn{2}{|c|}{ RMGI } & \multicolumn{2}{|c|}{ GIC } \\
\hline Discolora & ation & One surface & Two surfaces & One surface & Two surfaces \\
\hline Baseline & A & $31(100 \%)$ & $21(100 \%)$ & $22(100 \%)$ & $17(100 \%)$ \\
\hline \multirow[t]{2}{*}{ 6-Month } & $A$ & 29 (93.5\%) & $14(66.7 \%)$ & $21(95.5 \%)$ & $14(82.4 \%)$ \\
\hline & $\mathrm{B}$ & $2(6.5 \%)$ & $7(33.3 \%)$ & $1(4.5 \%)$ & $3(17.6 \%)$ \\
\hline \multirow[t]{4}{*}{ 12-Month } & $A$ & 27 (87.1\%) & $14(66.7 \%)$ & $18(85.7 \%)$ & $6(35.3 \%)$ \\
\hline & $\mathrm{B}$ & $4(12.9 \%)$ & 7 (33.3\%) & $1(4.8 \%)$ & $7(41.2 \%)$ \\
\hline & $\mathrm{C}$ & & & $2(9.5 \%)$ & $2(11.8 \%)$ \\
\hline & $\mathrm{D}$ & & & & $2(11.8 \%)$ \\
\hline \multirow[t]{4}{*}{ 24-Month } & $A$ & 27 (87.1\%) & $12(57.1 \%)$ & $17(81 \%)$ & $5(29.4 \%)$ \\
\hline & B & $4(12.9 \%)$ & $8(38.1 \%)$ & $1(4.8 \%)$ & $5(29.4 \%)$ \\
\hline & C & & $1(4.8 \%)$ & $3(14.3 \%)$ & $1(5.9 \%)$ \\
\hline & $\mathrm{D}$ & & & & $6(35.3 \%)$ \\
\hline \multicolumn{2}{|c|}{ Marginal } & \multicolumn{2}{|c|}{ RMGI } & \multicolumn{2}{|c|}{ GIC } \\
\hline Adaptatio & & One surface & Two surfaces & One surface & Two surfaces \\
\hline Baseline & $A$ & 31 (100\%) & $21(100 \%)$ & $22(100 \%)$ & $17(100 \%)$ \\
\hline \multirow{3}{*}{ 6-Month } & A & 29 (93.5\%) & $14(66.7 \%)$ & $18(81.8 \%)$ & $14(82.4 \%)$ \\
\hline & B & $2(6.5 \%)$ & 7 (33.3\%) & $4(18.2 \%)$ & $1(5.9 \%)$ \\
\hline & $\mathrm{C}$ & & & & \\
\hline \multirow{4}{*}{ 12-Month } & $A$ & 29 (93.5\%) & $11(52.4 \%)$ & $16(76.2 \%)$ & $6(35.3 \%)$ \\
\hline & $\mathrm{B}$ & $2(6.5 \%)$ & $10(47.6 \%)$ & $4(19 \%)$ & $5(29.4 \%)$ \\
\hline & $\mathrm{C}$ & & & $1(4.8 \%)$ & $4(23.5 \%)$ \\
\hline & $\mathrm{D}$ & & & & $2(11.8 \%)$ \\
\hline \multirow[t]{4}{*}{ 24-Month } & $A$ & 29 (93.5\%) & $10(47.6 \%)$ & $16(76.2 \%)$ & $5(29.4 \%)$ \\
\hline & $\mathrm{B}$ & $2(6.5 \%)$ & $10(47.6 \%)$ & $2(9.5 \%)$ & $3(17.6 \%)$ \\
\hline & C & & $1(4.8 \%)$ & $3(14.3 \%)$ & $3(17.6 \%)$ \\
\hline & $\mathrm{D}$ & & & & $6(35.3 \%)$ \\
\hline
\end{tabular}

$A=$ alpha, $B=$ bravo, $C=$ charlie, $D=$ delta $(p<0.05)$. 


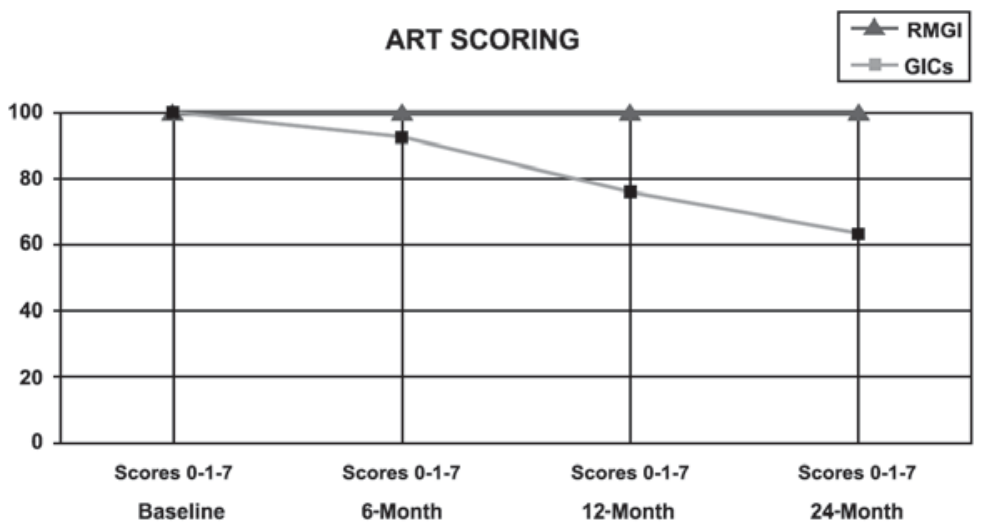

FIGURE 3- ART-scoring system success rate of GIC and RMGI material during the study period

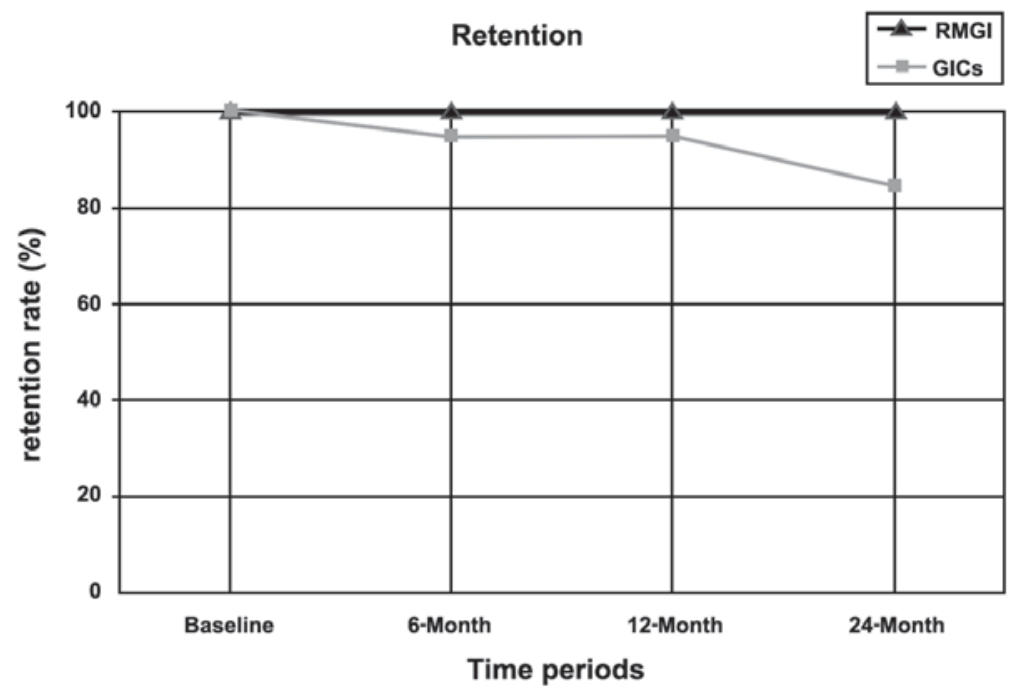

FIGURE 4- Retention rate of GIC and RMGI material during the study period

Contrary to RMGIC restorations, surface pitting at probing was the most common sign of surface loss in GIC group irrespective of single or multiple surface restorations. In comparison of both groups with respect to single surface restoration, there were statistically significant differences at each study periods in anatomic form category. However, the most pronounced difference was found at 24 month recall $(\mathrm{p}<0.001)$. For multiple surface restorations deteriorations in GIC restorations did increase with time, and it was statistically significant for all recall periods except of that in 6 months.

The null hypothesis that there is no difference in the success rate between restorations placed by the ART approach using HS-CICs and RMGICs after 2 years was thus rejected.

\section{DISCUSSION}

The results of this study showed that HSGIC restorations were limited as ART restoratives, especially in multiple surfaces. Also, RMGICs showed a better clinical performance than that of HSGICs, irrespective of the surface numbers for 24 months.

As seen in Table 1, total success rates of RMGIC for single- and multiple-surface restorations were 100 and $100 \%$, respectively. Corresponding values for HSGIC restorations were 80.9 and $41.2 \%$, respectively. Clearly, the $100 \%$ total retention rate of RMGIC materials with the ART technique is undoubtedly the greatest success rate ever seen. It has been demonstrated that the superior clinical performance of lightcured materials over those of chemical curing points out that they technically are less sensitive than self-cure materials because once irradiated, they do not require protection from moisture ${ }^{4}$.

Croll, et al. ${ }^{5}$ were the most enthusiastic of all the authors who generally support the use of RMGICs and light-curing HSGICs rather than those with chemical curing. In this respect, it could be argued that RMGIC materials with superior physical properties over GICs and controlled light curing can 
be recommended as an alternative material to be used for ART field trials. In parallel with this assumption, Donly, et al. ${ }^{6}$ concluded that in clinical terms RMGICs functioned as well as amalgam for Class II restorations in primary molars. Concomitantly, RMGICs exhibited significantly less enamel demineralization than amalgam at restoration margins, and, also, lower caries occurrence between tooth filling interfaces ${ }^{4,6}$. However, our 24-month results concerning both single-surface (100\%) and multiple-surface (100\%) RMGIC ART restorations $(100 \%)$ are very promising, with a success rate of over $98 \%$, which is the best retention rate reported until now for a two- year-follow up period.

The newer HSGICs marked specifically for the ART approach appear to be promising for the restoration of singlesurface-caries lesions in permanent teeth ${ }^{27}$. After 2 to 3 years, the success rate reported by most studies is approximately $90 \%$ for Class I, and Class-V-single-surface restorations; the rate for recurrent caries is approximately $2 \%$. One recent 3 year study reported higher survival rates for small restorations $(92 \%)$ than for large restorations $(77 \%)^{16}$. Studies involving earlier formulations of HSGIC showed slightly lower success rates (with a higher incidence of caries), as did restorations placed in children and in occlusal rather than nonocclusal surfaces $^{21,26}$. Statistically significant operator differences have also been reported. In present study, a success rate of $80.9 \%$ for single-surface-GI restorations seems to be lower than the recent two-year-studies by Ho, et al. ${ }^{15}$ in a clinical setting ( $89 \%$ and $93 \%$ for ChemFil Sup and Fuji IX, respectively), and by Mandari, et al. ${ }^{22}$ in a field setting (96\%). The reason for this low success rate as seen in the present study can be due to the participation of younger children, who are considered more difficult for restoring teeth; unavailability of a trained assistant used in many ART field trials to help the experienced dentist; and partially to the presence of large cavity size in many of the teeth restored ${ }^{19,23,28}$.

Very few studies have reported success rates for Class-IImulti-surface restorations in permanent teeth. After one year, one study of a HSGIC reported a success rate of $93 \%{ }^{3}$. Another recent study of Ketac-Molar found that eight out of 14 Class- II restorations were successful after 3 years $^{16}$. In general, the success rate of Class-II restorations after 24 months varied from 39.5 to $45.6 \%$ in both primary and permanent teeth, respectively. The 24-month-retention rate of multiple-surface-HSGIC restorations (totaling 41.2\%) is comparable to that of the permanent teeth.

Although the evidence supporting minimal restorative interventions resembling ART cavities shows that minute cavities can be successfully treated, material-related failures are not size dependent, and low-strength materials such as HSGICs seem to generally fail with respect to wear and tear in whatever cavity shape preferred ${ }^{28}$. Accordingly, in a recent study, fracturelmarginal defects and loss of material were among the most common reasons of failure in an ART group, even when the shape of the cavities had been modified. From these results, it may be concluded that the search of a new material that is stronger than HSGICs has become apparent ${ }^{22}$.

In some field trials, amalgam has been used as an ART restorative. In these studies, it has been clearly shown that amalgam is a suitable ART and/or manual restorative treatment (MRT) material for extensive occlusal lesions in high-caries populations, especially in young children; the secondary caries occurrence has been found to be significant when compared to HSGIC counterparts [2\% of glass-ionomer vs $10 \%$ of amalgam restorations] ${ }^{22}$. Ultimately, ART materials were used to remineralize the carious tissue remaining after hand excavation, and to prevent secondary caries ${ }^{8,25}$. In this respect, fluoride release and chemical adhesion is, of course, mandatory for future materials to be used in further ART studies, and clinical and field settings using adhesive materials with physical properties superior to the current HSGICs, such as compomers (polyacid modified composite resin) and RMGICs, would be meaningful. In a recent study, Ersin, et al. ${ }^{10}$ have tried to compare the effectiveness of a composite resin/dentin bonding system following an ART approach, as compared with HSGICs. Although their study was performed in primary dentition, after 2 years, the success rate of the composite material was found to be promising for further ART studies [96.7 percent of the Class-I-HSGIC restorations, and 91 percent of the resin-based composite restorations]. Clearly, the most remarkable, but not significant, result of this study was that the 24-month survival rate of the Class-II-composite restorations is much higher than that of HSGICs (82\% vs. $76.1 \%$ ) compared to the results seen in Class-II cavities. On the other hand, this logical application with composite resin according to an ART approach leads to a new chance of bonding the filling materials to the ART-hand-made cavities, using non-rinse-one-bottle bonding agents in field conditions.

One of the criteria used in the present study has been applied in most other ART studies in permanent dentitions ${ }^{11,16,18}$. However, the USPHS criteria are usually used to assess restoration survival in various studies. Holmgren, et al. ${ }^{16}$ applied both the ART and USPHS criteria to the same ART restorations in permanent teeth, and reported no significant differences in survival outcomes of ART restorations between the two sets of criteria. Lo, et al. ${ }^{20}$ suggested that the ART criteria are more stringent than the USPHS criteria. In the present study, we used both scoring systems including ART and USPHS criteria. The main reason was to compare the clinical performance of RMGIC material with the literature indicating a lack of previous reports on the issue of RMGIC with an ART approach. In only one 24month-study, the success rates observed using the USPHS criteria were close to, but lower than those observed using the ART criteria for two HSGIC restorative materials (98.1\% and $100 \%$, and $96.1 \%$ and $96.1 \%$ for Chem Flex and Fuji IX GP with USPHS and ART scoring criteria, respectively) ${ }^{19}$. This observation is in line with our study whereby, according to "retention criteria" of the USPHS system, the present results are higher than those obtained by the success-rate of ART (100\% and $80.9 \%$ with USPHS and ART, respectively) (Figures 3 and 4). It may reasonably be assumed that both criteria used in this study are to a large extent comparable to each other for evaluating ART fillings in field conditions. 


\section{CONCLUSION}

The results of the present study indicate that RMGIC cured by chargeable curing units would be able to provide a reliable alternative over glass ionomer materials in ART-field-trials. However, further clinical and field trials are needed to support this assumption.

\section{REFERENCES}

1- Barmes D. Foreword for proceedings of the IADR symposium minimal intervention techniques for dental caries. J Public Health Dent. 1996;56:1316.

2- Butani Y, Levy SM, Nowak AJ, Kanellis MJ, Heller K, Hartz AJ, et al. Overview of the evidence for clinical interventions in pediatric dentistry. Pediatr Dent. 2005;27:6-11.

3- Cefaly DF, Barata TJ, Bresciani E, Fagundes TC, Lauris JR, Navarro MF. Clinical evaluation of multiple-surface ART restorations: 12 month follow-up. J Dent Child. 2007;74:203-8

4- Croll TP, Bar-Zion Y, Segura A, Donly KJ. Clinical performance of resinmodified glass ionomer cement restorations in primary teeth: a retrospective evaluation. J Am Dent Assoc. 2001;132:1110-6.

5- Croll TP, Helpin ML, Donly KJ. Vitremer restorative cement for children: three clinicians' observations in three pediatric dental practices. ASDC J Dent Child. 2000;67:391-8.

6- Donly KJ, Segura A, Kanellis M, Erickson RL. Clinical performance and caries inhibition of resin-modified glass ionomer cement and amalgam restorations. J Am Dent Assoc. 1999;130:1459-66.

7- Dülgergil CT, Soyman M, Civelek A. Atraumatic restorative treatment with resin-modified glass ionomer material: short-term results of a pilot study. Med Princ Pract. 2005; 14:277-80.

8- Dutta BN, Gauba K, Tewari A, Chawla HS. Silver amalgam versus resin modified GIC class-II restorations in primary molars: twelve month clinical evaluation. J Indian Soc Pedod Prev Dent. 2001;19:118-22.

9- Eden E, Topaloglu Ak A, Frencken JE, van't Hof M. Survival of self-etch adhesive Class II composite restorations using ART and conventional cavity preparations in primary molars. Am J Dent. 2006;19:359-63.

10- Ersin NK, Candan U, Aykut A, Oncað O, Eronat C, Kose T. A clinical evaluation of resin-based composite and glass ionomer cement restorations placed in primary teeth using the ART approach: results at 24 months. J Am Dent Assoc. 2006;137:1529-36

11- Frencken JE, Makoni F, Sithole WD. ART restorations and glass-ionome sealants in Zimbabwe: survival after 3 years. Community Dent Oral Epidemiol. 1998;26:372-81.

12- Frencken JE, Pilot T, Songpaisan Y, Phantumvanit P. Atraumatic restorative treatment (ART): rationale, technique, and development. J Public Health Dent. 1996;56:135-40.

13- Frencken JE, Taifour D, van't Hof MA. Survival of ART and amalgam restorations in permanent teeth of children after 6.3 years. J Dent Res 2006;85:622-6.

14- Guggenberger R, May R, Stefan KP. New trends in glass-ionomer chemistry. Biomaterials. 1998;19:479-83.

15- Ho TF, Smales RJ, Fang DT. A 2-year clinical study of two glass ionome cements used in the atraumatic restorative treatment (ART) technique. Community Dent Oral Epidemiol. 1999;27:195-201.
16- Holmgren CJ, Lo EC, Hu D, Wan H. ART restorations and sealants placed in Chinese school children: results after three years. Community Dent Oral Epidemiol. 2000;28:314-20.

17- Holst A. A 3-year clinical evaluation of Ketac-Silver restorations in primary molars. Swed Dent J. 1996;20:209-14.

18- Lo EC, Holmgren CJ, Hu D, van Palenstein Helderman W. Six-year follow up of atraumatic restorative treatment restorations placed in Chinese school children. Community Dent Oral Epidemiol. 2007;35:387-92.

19- Lo EC, Luo Y, Fan MW, Wei SH. Clinical investigation of two glassionomer restoratives used with the Atraumatic Restorative Treatment approach in China: two-years results. Caries Res. 2001;35:458-63.

20- Lopez N, Simpser-Rafalin S, Berthold P. Atraumatic restorative treatment for prevention and treatment of caries in an underserved community. Am J Public Health. 2005;95:1338-9.

21- Mallow PK, Durward CS, Klaipo M. Restoration of permanent teeth in young rural children in Cambodia using the atraumatic restorative treatment (ART) technique and Fuji II glass ionomer cement. Int J Paediatr Dent. 1998:8:35-40.

22- Mandari GJ, Frencken JE, van't Hof MA. Six-year success rates of occlusal amalgam and glass-ionomer restorations placed using three minimal intervention approaches. Caries Res. 2003;37:246-53.

23- Mandari GJ, Truin GJ, van't Hof MA, Frencken JE. Effectiveness of three minimal intervention approaches for managing dental caries: survival of restorations after 2 years. Caries Res. 2001;35:90-4.

24- Monse-Schneider B, Heinrich-Weltzien R, Schug D, Sheiham A, Borutta A. Assessment of manual restorative treatment (MRT) with amalgam in high-caries Filipino children: results after 2 years. Community Dent Oral Epidemiol. 2003;31:129-35.

25- Palma-Dibb RG, Castro CG, Ramos RP, Chimello DT, Chinelatti MA. Bond strength of glass-ionomer cements to caries-affected dentin. J Adhes Dent. 2003;5:57-62.

26- Phantumvanit P, Songpaisan Y, Pilot T, Frencken JE. Atraumatic restorative treatment (ART): a three-year community field trial in Thailandsurvival of one-surface restorations in the permanent dentition. J Public Health Dent. 1996;56:141-5.

27- Smales RJ, Yip HK. The Atrauramatic Restorative Treatment (ART) approach for the management of the dental caries. Quintessence Int. 2002;33:427-32.

28- Walles AW, Wallwork MA, Holland IS, Murray JJ. The longevity of occlusal amalgam restorations in first permanent molars of child patients. Br Dent J. 1985;158:133-6.

29- Ziraps A, Honkala E. Clinical trial of a new glass ionomer for an atraumatic restorative treatment technique in class I restorations placed in Latvian school children. Med Princ Pract. 2002;11:44-7. 